\title{
A COMPLEMENT TO AUTOTUNING METHODS ON PID CONTROLLERS
}

\author{
R. Ferreiro García \& F.J. Pérez Castelo
}

Universidad de A Coruña. Dept. Ingeniería Industrial

E.S.M.C. Paseo de Ronda, 51, 15011 A Coruña, Spain

ferreiro@udc.es, javierpc@udc.es

Tel.: 3481167000 ext. 4205, Fax.: 3481167101

\begin{abstract}
This paper describes a complement to the autotuning ATV method. The proposed strategy is based in a frequency response design technique to get a three-term controller as a special case of a lead-lag design. The main topic of such autotuning technique concerns to the type of excitation signal on the plant which is a sine wave openloop excitation instead of a relay feedback used in the harmonic balance. Copyright 01999 IFAC
\end{abstract}

Keywords: ATV, FFT, Relay oscillation, Harmonic balance.

\section{INTRODUCTION AND PROBLEM STATEMENT}

The objective of autotuning methods is to obtain a PID controller capable for satisfying typical requirements such as rapid following, zero steady state error and overshoot suppression by means of a practical and robust method

The following methods are some of the most common methods to adjust the parameters of a PID controller:

- Relay feedback autotuning

- Frequency domain autotuning by magnitude and phase calculation

Relay feedback autotuning is used here to get the ultimate frequency. Such frequency is a reference to set the design operating frequency as a fraction of the reference one.

\subsection{Autotuning by Harmonic Balance method.}

Åstrom and Hagglund (1983) proposed an attractive method for determining the ultimate frequency and ultimate gain commonly called ATV (autotune variation). Such method consists in an approximative method (Åstrom and Hagglund 1983,1984, Åstrom \&
Wittenmark, 1989) called harmonic balance method based in relay feedback autotuning. Figure 1 shows a process transfer function with a feedback ideal relay. In parallel with a feedback relay there is a PID controller, the achieved virtual controller.

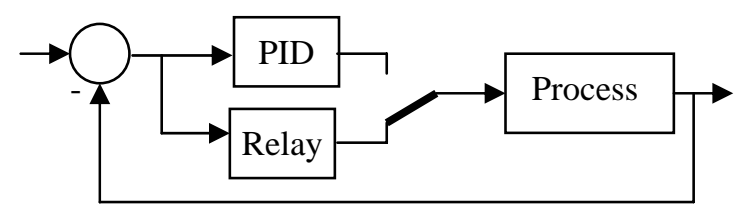

Fig. 1 Block diagram of a relay auto-tuner

An approximative condition for oscillation can be determined by assuming that there is a limit cycle with period $\mathrm{Tu}$ and frequency $\mathrm{w}_{\mathrm{u}}=2 \pi / \mathrm{Tu}$ such that the relay output is a periodic symmetric square wave. If the relay amplitude is $d$, a simple Fourier series expansion of the relay output shows that the first harmonic component has the amplitude $4 \mathrm{~d} / \pi$. Assume that the process dynamics correspond to a low-pass system and that the first harmonic contribution dominates the output. The error signal then has the amplitude

The condition for oscillation is given by 


$$
a=\frac{4 d}{\pi}|G(i W u)|
$$

The condition for oscillation is given by

$$
\arg G(i W u)=-\pi
$$

and the ultimate gain is achieved as

$$
K u=\frac{4 d}{\pi a}=\frac{1}{|G(i W u)|}
$$

where $\mathrm{Ku}$ can be regarded as the equivalent gain of the relay for transmission of sinusoidal signals with amplitude a. The parameters $\mathrm{d}$, a Tu are shown in Figure 2.

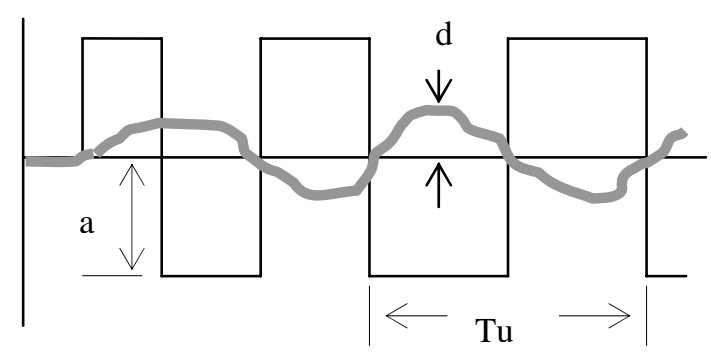

Fig. 2. Parameters of Harmonic Balance auto-tuner

So far only two parameters, $\mathrm{Ku}$ and $\mathrm{Tu}$ have been extracted from the relay experiment. With the data concerned to $\mathrm{Tu}$ and $\mathrm{Ku}$ it will now be shown how to determine the parameters of a PID controller. Table 1 shows the relationship between such parameters and the controller parameters given as $\mathrm{Kp}$, Ti and Td using the well-known method of Ziegler-Nichols tuning

Table 1. PID parameters

\begin{tabular}{lll}
\hline $\mathrm{Kp}$ & $\mathrm{Ti}$ & $\mathrm{Td}$ \\
\hline $0.6 . \mathrm{Ku}$ & $0.5 \mathrm{Tu}$ & $0.12 \mathrm{Tu}$ \\
$\mathrm{Kp}$ & $\mathrm{KI}$ & $\mathrm{KD}$ \\
$0.6 \mathrm{Ku}$ & $1.2 \mathrm{Ku} / \mathrm{Tu}$ & $0.07 \mathrm{Ku} . \mathrm{Tu}$ \\
\hline
\end{tabular}

At present developing state, an adaptive controller has been achieved by the ATV autotuning technique or relay feedback autotuning, which supply the frequency of the limit cycle and its associated gain, known as ultimate frequency and ultimate gain (Åstrom \& Wittenmark, 1989)

\subsection{Autotuning by frequency response method}

The method based in frequency response use a senoidal excitation instead of a relay excitation (Phillips and Nagle,1982). Figure 3 shows the structure of autotuning method

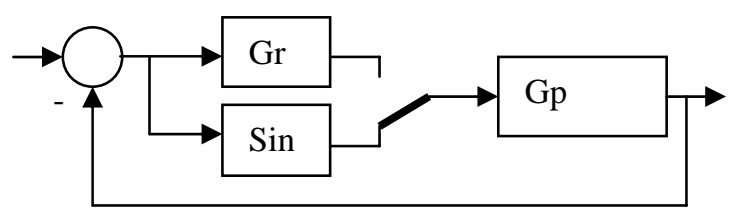

Fig. 3. Block diagram of a sine exciting auto-tuner

In this method the magnitude and phase at a given excitation frequency is to be determined. Typically, Fast Fourier Transform of the system response is used to achieve the magnitude and phase.

It is assumed, (Charles, Nagle, 1982) that a PID controller transfer function can be described as

$$
G r=K p+\frac{K_{I}}{w}+K_{D} w
$$

The controller frequency response is given by

$$
\begin{aligned}
& G r\left(j w_{W}\right)=K p+j\left(K_{D} w_{W}-\frac{K_{I}}{w_{W}}\right)= \\
& \left|G r\left(j w_{W}\right)\right| \angle \theta
\end{aligned}
$$

The design problem is to choose $\operatorname{Gr}(\mathrm{w})$, that is, to choose Kp, KI, KD such that

$$
G r\left(j w_{W 1}\right) G p\left(j w_{W 1}\right)=1 \angle\left(180+\phi_{m}\right)
$$

at a chosen frequency $\mathrm{w}_{\mathrm{w} 1}<\mathrm{w}_{\mathrm{u}}$, that is, a frequency $\mathrm{w}_{\mathrm{w} 1}$ lower that the ultimate frequency achieved by the relay oscillation autotuning method. Now, from equation (5)

$$
\begin{aligned}
& K p+j\left(K_{D} w_{W 1}-\frac{K_{I}}{w_{W}}\right)= \\
& \left|G r\left(j w_{W 1}\right)\right|(\cos \theta+j \sin \theta)= \\
& =\frac{\cos \theta}{\left|G p\left(j w_{W 1}\right)\right|}+j \frac{\sin \theta}{\left|G p\left(w_{W 1}\right)\right|}
\end{aligned}
$$

where

$$
\begin{aligned}
& \theta=180+\phi m-\angle\left(G p\left(j w_{W 1}\right)\right. \\
& K p=\frac{\cos \theta}{\left|G p\left(j w_{W 1}\right)\right|} \\
& K_{D} w_{W 1}-\frac{K_{I}}{w_{W 1}}=\frac{\sin \theta}{\left|G p\left(j w_{W 1}\right)\right|}
\end{aligned}
$$

The design equation is then described by equation (9). For a given plant $[\mathrm{Gp}(\mathrm{w})]$, the choice of $\mathrm{w}_{\mathrm{w} 1}$ and $\phi \mathrm{m}$ uniquely determines Kp. However, KI and KD are not uniquely determined. Satisfying equation (9), means that that any increment of KD will increase the bandwidth, while increments of KI, will decrease steady state errors for a given gain margin. 
Autotune methods has several advantages over openloop pulse testing methods:

- ATV method, no a priori knowledge of the system time constant is needed.

- Both methods, are closed loop tests, so the process will not drift away from the setpoint.

\section{ALTERNATIVE DESIGN METHOD}

The proposed autotune method is based in the type of excitation signal on the plant, which is a senoidal openloop excitation signal instead of a relay feedback.

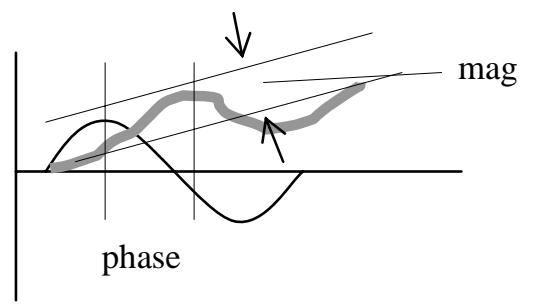

Fig 4. Time response of a sine wave excitation

The main advantage of this method is that only a sine wave period is applied to excite the plant instead of several cycles as in the FFT method.

The key idea is such that the time response of the sine wave excitation signal is analysed to achieve the magnitude and phase as shown in Figure 4 instead frequency response by FFT computation, as well as in the relay feedback method.

Design equations are achieved after some manipulation of the design method described by equation (9). So that,

$$
\begin{aligned}
& K p=\frac{\cos \theta}{\left|G p\left(j w_{W 1}\right)\right|} \\
& K p \cdot T_{D} w_{W 1}-\frac{K p \cdot T_{I}}{w_{W 1}}=\frac{\sin \theta}{\left|G p\left(j w_{W 1}\right)\right|}
\end{aligned}
$$

and finally

$$
\begin{aligned}
& K p=\frac{\cos \theta}{\left|G p\left(j w_{W 1}\right)\right|} \\
& T_{D} w_{W 1}-\frac{T_{I}}{w_{W 1}}=\tan \theta
\end{aligned}
$$

At this state of the analysis, a criterion is needed to solve the problem of determining the values of $\mathrm{Kp}$, TI and TD. It is proposed a parameter value $\beta$ that relates the parameter TI with TD as,

$$
T_{I}=\beta \cdot T_{D}
$$

With this proposition, expression (11) yields

$$
\begin{aligned}
& K p=\frac{\cos \theta}{\left|G p\left(j w_{W 1}\right)\right|} \\
& T_{I}=\beta \cdot T_{D} \\
& T_{D}=\frac{\tan \theta}{2 \cdot w_{W 1}} \pm \frac{1}{w_{W 1}} \sqrt{\frac{\tan ^{2} \theta}{4}+\frac{1}{\beta}}
\end{aligned}
$$

Under Ziegler \& Nichols criteria, $\beta=4$ and hence

$$
\begin{aligned}
& K p=\frac{\cos \theta}{\left|G p\left(j w_{W 1}\right)\right|} \\
& T_{I}=4 \cdot T_{D} \\
& T_{D}=\frac{1}{2 \cdot w_{W 1}}\left(\tan \theta \pm \sqrt{\tan ^{2} \theta+1}\right)
\end{aligned}
$$

or, after some manipulation

$$
\begin{aligned}
& K p=\frac{\cos \theta}{\left|G p\left(j w_{W 1}\right)\right|} \\
& T_{I}=4 \cdot T_{D} \\
& T_{D}=\frac{\sin \theta+1}{2 \cdot w_{W 1} \cos \theta}
\end{aligned}
$$

where in most of industrial process control applications, for $\beta=4$ successful results are achieved.

If, with the selected exciting frequency and phase margin, achieved autotune values are not satisfactory, then, another frequency lower than the one selected is to be applied.

\section{APPLICATION OF THE METHOD}

An object oriented tool had been developed under HP.VEE environment which can operate alternatively off-line by simulation of a modelled process or on-line connected to the process in real time operation. The main goal of such a tool is the possibility of being used in a pre-tuning task by using an approach to the plant model.

The algorithmic procedure consists of the following phases:

- Excitation of the plant with a complete sine wave period of the frequency at which the controlled plant will operate $\mathrm{w}_{\mathrm{w} 1}$ 
- Measuring the magnitude and phase by time analysis.

- $\quad$ Apply the expressions (8) and (15).

to achieve the regulator parameters while satisfying a desired phase margin $\phi \mathrm{m}$. at an operating frequency.

\section{RESULTS AND CONCLUSIONS}

The proposed method (Time or frequency response analysis =FRA) is compared with the method of (Harmonic Balance $=\mathrm{HB}$ ). Comparison is performed by application of the benchmark system for PID control (Åstrom and Hägglund, 2000) on system one with $\mathrm{n}=2$ in table 2 and $\mathrm{n}=3$ in table 3 . Operation excitation frequency has been selected as $0.2 \mathrm{cps}$ for $\mathrm{n}=2$ system and 0.3 cps for $\mathrm{n}=3$ system.

Table 2. Autotuning results with $\mathrm{n}=2$ and $0.2 \mathrm{cps}$

\begin{tabular}{lll}
\hline & HB & FRA \\
\hline Kp & 15.4 & 2.4 \\
$\mathrm{Ti}$ & 0.64 & 6 \\
$\mathrm{Td}$ & 0.16 & 1.5 \\
\hline
\end{tabular}

Table 3. Autotuning results with $\mathrm{n}=3$ and $0.3 \mathrm{cps}$

\begin{tabular}{lll}
\hline & HB & FRA \\
\hline $\mathrm{Kp}$ & 3.3 & 4.3 \\
$\mathrm{Ti}$ & 2.2 & 13.3 \\
$\mathrm{Td}$ & 0.54 & 3.3 \\
\hline
\end{tabular}

The Tables 2 and 3 show the corresponding PID parameters obtained for both systems with both methods. The system identification is represented in Figures 4 and 5 and the system response in Figure 6.

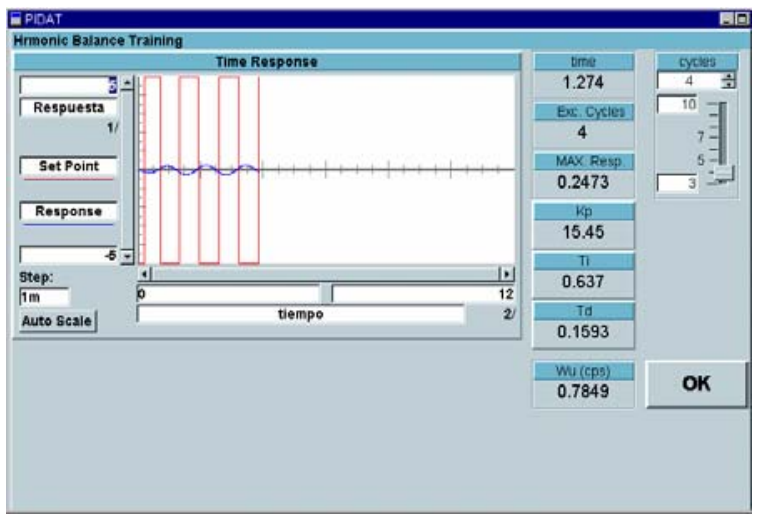

Fig. 4. HB identification for system 1 and $n=2$

Another topic in a pre-tuning task is the possibility of using this method as pre-tune values or use the averaged parameters of alternative tuning methods. In any case, the possibility of comparing the results of several methods increases the reliability of the autotuning test.

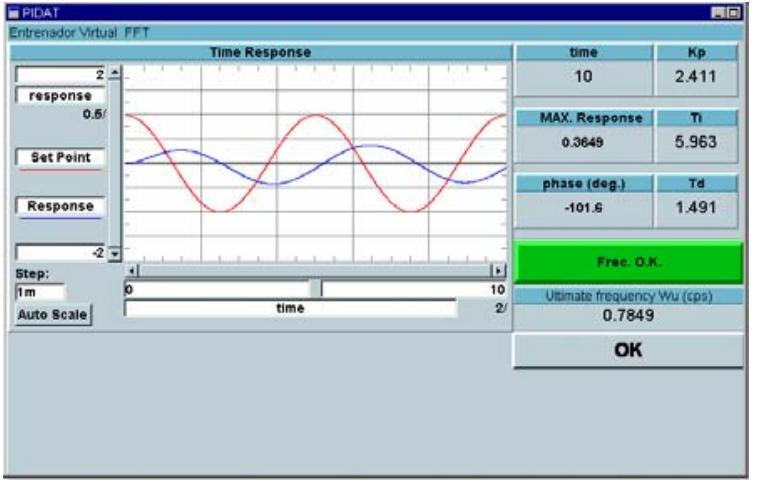

Fig5. FRA identification for system 1 and $n=2$

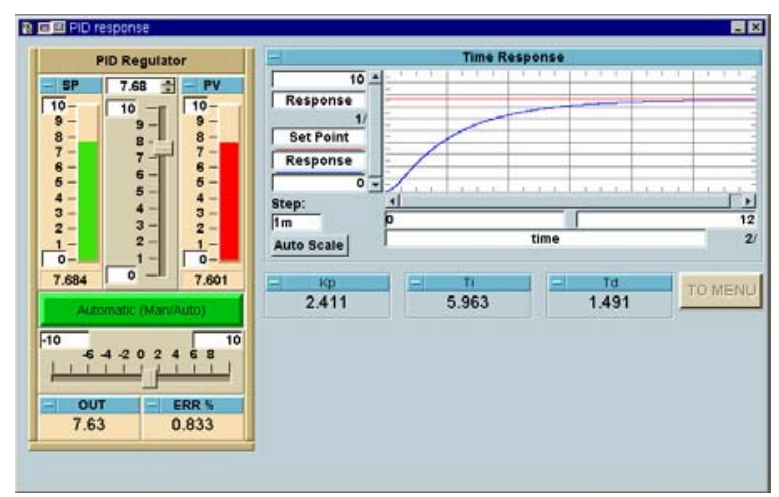

Fig 6. System 1 time response with PID designed with FRA method.

As a general criterion, it can be assumed that the appropriate excitation frequency will be the maximum value for which the controlled system responds properly under a desired phase margin. There is a disadvantage in the proposed method concerning to the need of some a priori knowledge about the frequency at which the plant could respond properly. A trial and error procedure is a practical way to find this frequency.

\section{REFERENCES}

Åstrom and Hagglund (1983).Proceedings of IFAC Conference. San Francisco. USA.

Åstrom and Hagglund (1984). Automatic tuning of simple regulators with specifications on phase and amplitude margins. Automatica Vol 20, pp 645-651

Åstrom and Bjorn Wittenmark (1989). Adaptive Control, Chap. 8, pp 332-339. Ed. Addison Wesley publishing Company. USA

Åstrom and Hägglund (2000) Benchmark Systerms for PID Control. PID’00,IFAC Workshop on Digital Control. Terrassa Spain

Charles L. Phillips, H. Troy Nagle, J.R, (1982) Digtal Control Systems. Analysis and Design. Chap.8,pp 254-258. Ed. Prentice Hall. Englewood Cliffs. N.J. 07632. USA 